\title{
THE ZAPPA-SZEP PRODUCT OF LEFT-ORDERABLE GROUPS
}

\author{
FABIENNE CHOURAQUI
}

\begin{abstract}
It is well-known that the direct product of left-orderable groups is leftorderable and that, under a certain condition, the semi-direct product of left-orderable groups is left-orderable. We extend this result and show that, under a similar condition, the Zappa-Szep product of left-orderable groups is left-orderable. Moreover, we find conditions that ensure the existence of a partial left and right invariant ordering (bi-order) in the Zappa-Szep product of bi-orderable groups and prove some properties satisfied.
\end{abstract}

\section{INTRODUCTION}

Let $G$ be a group with subgroups $H$ and $K$ such that $G=H K$ and $H \cap K=\{1\}$. Then $G$ is isomorphic to the Zappa-Szep product of $H$ and $K$, denoted by $H \bowtie K$. If both subgroups $H$ and $K$ are normal, $G$ is their direct product $H \times K$ and if $H$ only is normal, $G$ is their semi-direct product $H \rtimes K$. The Zappa-Szep product of groups is a generalisation of the direct and the semi-direct product which requires the embedding of neither of the factors to be normal in the product. We recall that a group $G$ is left-orderable if there exists a total ordering $\prec$ of its elements which is invariant under left multiplication, that is $g \prec h$ implies $f g \prec f h$ for all $f, g, h$ in $G$. It is well-known that the direct product of left-orderable groups is left-orderable and that, under a certain condition, the semi-direct product of left-orderable groups is left-orderable [9, [8] [p.27]. We extend this result and show that, under a similar condition, the Zappa-Szep product of left-orderable groups is left-orderable. Before we state our first result, we introduce some notations.

Definition. Let $H, K$ be groups. Let $\alpha$ be the homomorphism defined by $\alpha: K \rightarrow \operatorname{Sym}(H)$, $k \mapsto \alpha_{k}$, where $k \in K$ and $\operatorname{Sym}(H)$ denotes the group of bijections of $H$. Let $\beta: H \rightarrow$ $\operatorname{Sym}(K), h \mapsto \beta_{h}$, be such that $\beta_{h_{1} h_{2}}=\beta_{h_{2}} \circ \beta_{h_{1}}$ and $\beta_{1}=I d_{K}$ ( $\beta$ an anti-homomorphism). Assume $(\alpha, \beta)$ satisfies: $\alpha_{k}\left(h_{1} h_{2}\right)=\alpha_{k}\left(h_{1}\right) \alpha_{\beta_{h_{1}}(k)}\left(h_{2}\right)$ and $\beta_{h}\left(k_{1} k_{2}\right)=\beta_{\alpha_{k_{2}}(h)}\left(k_{1}\right) \beta_{h}\left(k_{2}\right)$. The Zappa-Szep product $H \bowtie K$ of $H$ and $K$ with respect to the pair $(\alpha, \beta)$ is the set $H \times K$ endowed with the following product:

$$
\left(h_{1}, k_{1}\right)\left(h_{2}, k_{2}\right)=\left(h_{1} \alpha_{k_{1}}\left(h_{2}\right), \beta_{h_{2}}\left(k_{1}\right) k_{2}\right)
$$

The identity is $(1,1)$ and the inverse of an element $(h, k)$ is

$$
(h, k)^{-1}=\left(\alpha_{k^{-1}}\left(h^{-1}\right), \beta_{h^{-1}}\left(k^{-1}\right)\right)
$$

In fact, $H \times\{1\}$ and $K \times\{1\}$ are subgroups of $H \bowtie K$, isomorphic to $H$ and $K$, respectively.

The Zappa-Szep product is also called crossed product, bi-crossed product, knit product, two-sided semidirect product. We refer to [3], [1], [13, [14] for details. Note that every element in $H \bowtie K$ is uniquely represented by $h k$, with $h \in H$ and $k \in K$, and $k h$ is equal to $\alpha_{k}(h) \beta_{h}(k)$ in $H \bowtie K$.

Theorem 1. Let $G$ be the Zappa-Szep product of the groups $H$ and $K$ with respect to $(\alpha, \beta)$. Assume $H$ and $K$ are left-orderable groups. Let $P_{H}$ and $P_{K}$ denote the positive cones (of a left order) of $H$ and $K$ respectively. Assume the following condition $(*)$ is satisfied:

$$
\alpha_{k}\left(P_{H}\right) \subseteq P_{H}, \forall k \in K
$$


Then, there exists a total left order $\prec$ on $G=H \bowtie K$, with positive cone $P$, such that (the embedding of ) $K$ is a convex subgroup with respect to $\prec$ and $P_{K}=P \cap K$.

We introduce some definitions and refer to $[2,6,7,6,9,10,11,12$. Let $G$ be a left-orderable group with a strict total left order $\prec$. An element $g, g \in G$, is called positive if $1 \prec g$ and the set of positive elements $P$ is called the positive cone of $\prec$. The positive cone $P$ satisfies:

(1) $P$ is a semigroup, that is $P \cdot P \subseteq P$

(2) $G$ is partitioned, that is $G=P \cup P^{-1} \cup\{1\}$ and $P \cap P^{-1}=\emptyset$

Conversely, if there exists a subset $P$ of $G$ that satisfies (1) and (2), then $P$ determines a unique total left order $\prec$ defined by $g \prec h$ if and only if $g^{-1} h \in P$. If $P$ satisfies only (1), the left order obtained is partial. A subgroup $N$ of a left-orderable group $G$ is called convex if for any $x, y, z \in G$ such that $x, z \in N$ and $x \prec y \prec z$, we have $y \in N$.

Proof. of Theorem 1 Let $P \subseteq G$ be defined by: $g=h k \in P$ if $h \in P_{H}$ or if $h=1, k \in P_{K}$. We show there exists a total left order $\prec$ on $G=H \bowtie K$ with positive cone $P$. First, we prove $P$ is a semigroup. Let $g=h k \in P$ and $g^{\prime}=h^{\prime} k^{\prime} \in P$. If $h^{\prime}=1$ and $h=1$, then $k, k^{\prime} \in P_{K}$ and $k k^{\prime} \in P_{K}$, since $P_{K}$ is a semigroup, so $g g^{\prime} \in P$. If $h^{\prime}=1$ and $h \neq 1$, then $h \in P_{H}$ and so $g g^{\prime}=h k k^{\prime} \in P$. If $h^{\prime} \neq 1$, then $h^{\prime} \in P_{H}$ and $g g^{\prime}=h k h^{\prime} k^{\prime}=h \alpha_{k}\left(h^{\prime}\right) \beta_{h^{\prime}}(k) k^{\prime}$. From the assumption $(*), \alpha_{k}\left(h^{\prime}\right) \in P_{H}$, so if $h=1$, then $g g^{\prime}=\alpha_{k}\left(h^{\prime}\right) \beta_{h^{\prime}}(k) k^{\prime} \in P$ and if $h \neq 1, h \alpha_{k}\left(h^{\prime}\right) \in P_{H}$ also, since $P_{H}$ is a semigroup, so $g g^{\prime} \in P$. Next, we prove that given $g=h k \neq 1$ in $G=H \bowtie K$, either $g$ belongs to $P$ or $g^{-1}=\alpha_{k^{-1}}\left(h^{-1}\right) \beta_{h^{-1}}\left(k^{-1}\right)$ belongs to $P$. Assume $h=1$. If $k \in P_{K}$, then $g \in P$, otherwise $k^{-1} \in P_{K}$, since $P_{K}$ partitions $K$, and then $g^{-1}=k^{-1} \in P$. Furthermore, $g$ cannot belong to $P \cap P^{-1}$, since it would contradict $P_{K} \cap P_{K}^{-1}=\emptyset$. Assume $h \neq 1$. If $h \in P_{H}$, then $g \in P$, otherwise $h^{-1} \in P_{H}$, since $P_{H}$ partitions $H$, and from $(*), \alpha_{k^{-1}}\left(h^{-1}\right) \in P_{H}$. So, $g^{-1}=\alpha_{k^{-1}}\left(h^{-1}\right) \beta_{h^{-1}}\left(k^{-1}\right)$ belongs to $P$. Assume $g \in P \cap P^{-1}$, then $h \in P_{H}$ and $\alpha_{k^{-1}}\left(h^{-1}\right) \in P_{H}$. It holds that $\alpha_{k^{-1}}\left(h^{-1}\right)=\left(\alpha_{\beta_{h^{-1}}\left(k^{-1}\right)}(h)\right)^{-1}$. Indeed, on one side $\alpha_{k^{-1}}(1)=1$ and on the other side $\alpha_{k^{-1}}(1)=\alpha_{k^{-1}}\left(h^{-1} h\right)=\alpha_{k^{-1}}\left(h^{-1}\right) \alpha_{\beta_{h^{-1}}\left(k^{-1}\right)}(h)$. From $(*), \alpha_{\beta_{h^{-1}}\left(k^{-1}\right)}(h)$ belongs to $P_{H}$, so $\alpha_{k^{-1}}\left(h^{-1}\right)=\left(\alpha_{\beta_{h-1}\left(k^{-1}\right)}(h)\right)^{-1}$ belongs to $P_{H} \cap P_{H}^{-1}$ and this is a contradiction. So, $P$ satisfies the conditions (1) and (2) and it determines uniquely a total left order $\prec$.

Let $g=h k \in G$ and $k^{\prime} \in K$. Assume $1 \prec g \prec k^{\prime}$ and assume by contradiction that $h \neq 1$. From $1 \prec g$, we have $h \in P_{H}$. From $h k \prec k^{\prime}$, we have $k^{\prime-1} h k \prec 1$. But, $k^{\prime-1} h k=\alpha_{k^{\prime-1}}(h) \beta_{h}\left(k^{\prime-1}\right) k \succ 1$, since, from $(*), \alpha_{k^{\prime-1}}(h)$ belongs to $P_{H}$. So, $h=1$.

In the following example, we remark it is sometimes useful to consider a semi-direct product as a special case of Zappa-Szep product in order to show it is left-orderable. Indeed, this permits to check condition (*) either on a positive cone of $H$ or on a positive cone of $K$. Let $H=\operatorname{Gp}\left\langle y, z \mid y^{3}=z^{3}\right\rangle$. Since $H=\mathbb{Z} *_{3 \mathbb{Z}} \mathbb{Z}$ and $\mathbb{Z}$ is (left) orderable, $H$ is left-orderable 8 [p.178]. Let $K=\operatorname{Gp}\langle a, b \mid a b a=b a b\rangle$, the braid group on 3 strands, $K$ is left-orderable [5. Let $\alpha: K \rightarrow \operatorname{Sym}(H)$ be the trivial homomorphism and let $\beta: H \rightarrow \operatorname{Sym}(K)$, the anti-homomorphism defined by $\beta_{y}=\beta_{z}=(a, b)$. With respect to $(\alpha, \beta)$, let $G=H \bowtie K$ in this specific order; $G$ is presented by $\mathrm{Gp}\langle y, z, a, b| a b a=b a b, y^{3}=z^{3}, a y=y b, b y=$ $y a, a z=z b, b z=z a\rangle$. We show $G$ is left-orderable, using Thm 1. Each element $h \in H$ admits a normal formal $h=y^{\epsilon_{1}} z^{\mu_{1}} y^{\epsilon_{2}} z^{\mu_{2}} \ldots y^{\epsilon_{m}} z^{\mu_{m}} \Delta^{n_{h}}$, where $\Delta=y^{3}=z^{3}$ is central in $H,-1 \leq \epsilon_{i}, \mu_{i} \leq 1$ and $n_{h} \in \mathbb{Z}$. We define $\exp (h)=\epsilon_{1}+\mu_{1}+. .+\epsilon_{m}+\mu_{m}+3 n_{h}$ and $P_{H}$ to be the set of elements $h \in H \backslash\{1\}$ such that $\exp (h)>0$, or if $\exp (h)=0, n_{h}>0$, or if $\exp (h)=n_{h}=0, \epsilon_{1} \neq 0$. The set $P_{H}$ is a positive cone (the proof appears in the appendix) and $P_{H}$ satisfies trivially the condition (*) from Thm 1 So, $G$ is left-orderable.

Note that a group is left-orderable if and only if it is right-orderable. This is well illustrated here with the existence of a symmetric version of this result. Indeed, if $H$ and $K$ are right-orderable groups, with $Q_{H}$ and $Q_{K}$ positive cones of right orders of $H$ and $K$ 
satisfying $\beta_{h}\left(Q_{K}\right) \subseteq Q_{K}, \forall h \in H$. Then there exists a total right order $<$ on $G=H \bowtie K$, with positive cone $Q$ (defined by $g=h k \in Q$ if $k \in Q_{K}$ or if $k=1, h \in Q_{H}$ ), such that (the embedding of ) $H$ is a convex subgroup with respect to $<$. We say a group $G$ is partially (totally) bi-orderable if there exists a partial (total) ordering $\ll$ of its elements which is invariant under left and right multiplication, that is $g \ll h$ implies $f g k \ll f h k$ for all $f, g, h, k$ in $G$. In particular, a set $P$ determines a partial bi-order if and only if $P$ is a semigroup, and satisfies $g P g^{-1} \subseteq P$ for all $g \in G$; $P$ determines a total bi-order if additionally $G=P \cup P^{-1} \cup\{1\}$ and $P \cap P^{-1}=\emptyset$. A natural question is when the Zappa-Szep product of bi-orderable groups is a bi-orderable group. In the following theorem, we give conditions that ensure the existence of a partial bi-order.

Theorem 2. Let $G$ be the Zappa-Szep product of the groups $H$ and $K$ with respect to $(\alpha, \beta)$. Assume $H$ and $K$ are partially bi-orderable groups. Let $P_{H}$ and $P_{K}$ denote the positive cones (of a partial bi-order) of $H$ and $K$ respectively. Assume the following conditions are satisfied: (*) $\alpha_{k}\left(P_{H}\right) \subseteq P_{H}, \forall k \in P_{K}$, and $\beta_{h}\left(P_{K}\right) \subseteq P_{K}, \forall h \in P_{H}$

(**) $k P_{H} k^{-1} \subseteq P_{H}, \forall k \in K$, and $h P_{K} h^{-1} \subseteq P_{K}, \forall h \in H$

Then, there exists a partial bi-order $\ll$ on $G=H \bowtie K$. Furthermore, if $h, h^{\prime} \in H$ satisfy $h \ll h^{\prime}$ then $\alpha_{k}(h) \ll \alpha_{k}\left(h^{\prime}\right)$ and $\beta_{h}(k) \ll \beta_{h^{\prime}}(k), \forall k \in K$ and if $k, k^{\prime} \in K$ satisfy $k \ll k^{\prime}$ then $\alpha_{k}(h) \ll \alpha_{k^{\prime}}(h)$ and $\beta_{h}(k) \ll \beta_{h}\left(k^{\prime}\right), \forall h \in H$.

Proof. Let $P \subseteq G$ be defined by: $g=h k \in P$ if $h \in P_{H}$ and $k \in P_{K}$; if $h=1, k \in P_{K}$ or if $k=1, h \in P_{H}$. We show $P$ is a semigroup and $g P g^{-1} \subseteq P$ for all $g \in G$. Let $g=h k \in P$ and $g^{\prime}=h^{\prime} k^{\prime} \in P$, then $g g^{\prime}=h k h^{\prime} k^{\prime}=h \alpha_{k}\left(h^{\prime}\right) \beta_{h^{\prime}}(k) k^{\prime}$. From the assumption (*), $\alpha_{k}\left(h^{\prime}\right) \in P_{H}$ and $\beta_{h^{\prime}}(k) \in P_{K}$, so $h \alpha_{k}\left(h^{\prime}\right) \in P_{H}$ and $\beta_{h^{\prime}}(k) k^{\prime} \in P_{K}$, since $P_{H}$ and $P_{K}$ are semigroups, so $g g^{\prime} \in P$. Next, let $g=h k \in G$ and $h^{\prime} k^{\prime} \in P$, we show $g h^{\prime} k^{\prime} g^{-1} \in P$ : $g h^{\prime} k^{\prime} g^{-1}=h k h^{\prime} k^{\prime} k^{-1} h^{-1}=\left(h\left(k h^{\prime} k^{-1}\right) h^{-1}\right)\left(h\left(k k^{\prime} k^{-1}\right) h^{-1}\right)$. From $(* *), h^{\prime} \in P_{H}$ implies $k h^{\prime} k^{-1} \in P_{H}$ and so $h\left(k h^{\prime} k^{-1}\right) h^{-1} \in P_{H}$, since $P_{H}$ is the positive cone of a bi-order. The element $h\left(k k^{\prime} k^{-1}\right) h^{-1} \in P_{K}$, first from $k k^{\prime} k^{-1} \in P_{K}$, and next from $(* *)$.

Let $h, h^{\prime} \in H$ satisfy $h \ll h^{\prime}$, and let $k \in K$, then $k h \ll k h^{\prime}$, that is $\alpha_{k}(h) \beta_{h}(k) \ll$ $\alpha_{k}\left(h^{\prime}\right) \beta_{h^{\prime}}(k)$. Since $\ll$ is a bi-order, this implies $1 \ll\left(\alpha_{k}(h)\right)^{-1} \alpha_{k}\left(h^{\prime}\right) \beta_{h^{\prime}}(k)\left(\beta_{h}(k)\right)^{-1}$ and from the definition of $\ll$, we have $\left(\alpha_{k}(h)\right)^{-1} \alpha_{k}\left(h^{\prime}\right) \in P_{H}$ and $\beta_{h^{\prime}}(k)\left(\beta_{h}(k)\right)^{-1} \in P_{K}$, that is $\alpha_{k}(h) \ll \alpha_{k}\left(h^{\prime}\right)$ and $\beta_{h}(k) \ll \beta_{h^{\prime}}(k)$. In case $h=1 h^{\prime} \neq 1$, this means $\alpha_{k}\left(P_{H}\right) \subseteq P_{H}, \forall k \in$ $K$ and $h^{\prime} \gg 1$ implies $\beta_{h^{\prime}}(k) \gg k, \forall k \in K$; in case $h \neq 1 h^{\prime}=1$, this means that $h \ll 1$ implies $\alpha_{k}(h) \ll 1$ and $\beta_{h}(k) \ll k, \forall k \in K$. The same proof works for the symmetric statement.

It would be interesting to know if there exists a natural extension of total bi-orders of $H$ and $K$ that defines a total bi-order on $H \bowtie K$ and $H \bowtie K$ is not a direct nor a semi-direct product.

\section{APPENDIX}

We recall each element $h \in H$ admits a normal formal $h=y^{\epsilon_{1}} z^{\mu_{1}} y^{\epsilon_{2}} z^{\mu_{2}} \ldots y^{\epsilon_{m}} z^{\mu_{m}} \Delta^{n_{h}}$, where $\Delta=y^{3}=z^{3}$ is a central element in $H,-1 \leq \epsilon_{i}, \mu_{i} \leq 1$ and $n_{h} \in \mathbb{Z}$. We define $\exp (h)=\epsilon_{1}+\mu_{1}+. .+\epsilon_{m}+\mu_{m}+3 n_{h}$ and $P_{H}$ to be the set of elements $h \in H \backslash\{1\}$ such that $\exp (h)>0(\operatorname{class}(\mathrm{A}))$, or if $\exp (h)=0, n_{h}>0$ (class (B)), or if $\exp (h)=n_{h}=0$, $\epsilon_{1} \neq 0$ (class $(\mathrm{C})$ ). We show $P_{H}$ is a positive cone. First, we show $P_{H}$ is a semigroup. Let $h=y^{\epsilon_{1}} z^{\mu_{1}} \ldots y^{\epsilon_{m}} z^{\mu_{m}} \Delta^{n_{h}}, h^{\prime}=y^{\epsilon_{1}^{\prime}} z^{\mu_{1}^{\prime}} \ldots y^{\epsilon_{m}^{\prime}} z^{\mu_{m}^{\prime}} \Delta^{n_{h^{\prime}}}$, with $-1 \leq \epsilon_{i}, \mu_{i}, \epsilon_{i}^{\prime}, \mu_{i}^{\prime} \leq 1$, be elements of $P_{H}$. We need to check several cases in order to show $h h^{\prime} \in P_{H}$.

Case 1: if $h$ is in class (A) and $h^{\prime}$ is in class (A) or (B) or (C), then $h h^{\prime}$ is also in (A).

Case 2: if $h, h^{\prime}$ are in class (B), then $h h^{\prime} \in P_{H}$, since $\exp \left(h h^{\prime}\right)=0$ and $n_{h h^{\prime}} \geq n_{h}+n_{h^{\prime}}-1>0$. Indeed, there are only two cases in which $n_{h h^{\prime}}$ decreases: if $h$ ends with $y^{-1}$ and $h^{\prime}$ begins with $y^{-1}$, or if $h$ ends with $z^{-1}$ and $h^{\prime}$ begins with $z^{-1}$, and in both cases $n_{h h^{\prime}}=n_{h}+n_{h^{\prime}}-1$. 
Case 3: assume $h, h^{\prime}$ are in class (C). Note that if $h=y^{\epsilon_{1}} z^{\mu_{1}} \ldots y^{\epsilon_{m}} z^{\mu_{m}} \Delta^{n_{h}},-1 \leq \epsilon_{i}, \mu_{i} \leq 1$, satisfies $\exp (h)=n_{h}=0$, then the length of $h$ is necessarily even. Furthermore, if $h$ is in class (C), then $h=y^{ \pm 1} z^{\mu_{1}} y^{\epsilon_{2}} z^{\mu_{2}} \ldots y^{\epsilon_{m}} z^{ \pm 1}$ and the same holds for $h^{\prime}$. So, $h h^{\prime}$ is also in (C). Case 4: if $h$ is in class (B) and $h^{\prime}$ is in class (C), then $h=y^{\epsilon_{1}} z^{\mu_{1}} \ldots y^{\epsilon_{m}} z^{\mu_{m}} \Delta^{n_{h}}, n_{h}>0$ and $h^{\prime}=y^{ \pm 1} z^{\mu_{1}^{\prime}} \ldots y^{\epsilon_{m}^{\prime}} z^{ \pm 1}$. It holds that $n_{h}-1 \leq n_{h h^{\prime}} \leq n_{h}+1$ and we need only to check the case $n_{h h^{\prime}}=n_{h}-1$. If $n_{h}-1>0$, then $h h^{\prime}$ is in class (B). If $n_{h}-1=0$, then $h=y^{\epsilon_{1}} z^{\mu_{1}} \ldots z^{\mu_{m-1}} y^{-1} \Delta, h^{\prime}=y^{-1} z^{\mu_{1}^{\prime}} \ldots y^{\epsilon_{m}^{\prime}} z^{ \pm 1}$ and $h h^{\prime}=y^{\epsilon_{1}} z^{\mu_{1}} \ldots z^{\mu_{m-1}} y z^{\mu_{1}^{\prime}} \ldots y^{\epsilon_{m}^{\prime}} z^{ \pm 1}$. We show $h h^{\prime}$ is in class $(\mathrm{C})$, that is $\epsilon_{1} \neq 0$. On one-hand, $\exp (h)=\epsilon_{1}+\mu_{1}+\ldots+\mu_{m-1}-1+3$ and on the second-hand $\exp (h)=0$. By induction on $m$, it holds that $\epsilon_{1}+\mu_{1}+\ldots+\epsilon_{m-1}+$ $\mu_{m-1}=-2$, with $-1 \leq \epsilon_{i}, \mu_{i} \leq 1$, implies $\epsilon_{1} \neq 0$.

Case 5: if $h$ is in class (C) and $h^{\prime}$ is in class (B), then $h=y^{ \pm_{1}} z^{\mu_{1}} \ldots y^{\epsilon_{m}} z^{ \pm 1}$, and $h^{\prime}=$ $y^{\epsilon_{1}^{\prime}} z^{\mu_{1}^{\prime}} \ldots y^{\epsilon_{m}^{\prime}} z^{\mu_{m}^{\prime}} \Delta^{n_{h^{\prime}}}, n_{h^{\prime}}>0$. It holds that $n_{h^{\prime}}-1 \leq n_{h h^{\prime}} \leq n_{h^{\prime}}+1$ and in case $n_{h h^{\prime}}=$ $n_{h^{\prime}}-1=0, h h^{\prime}$ is necessarily in class $(\mathrm{C})$, as $h h^{\prime}$ begins with $y^{ \pm 1}$.

Next, we show $P_{H}$ partitions $H$, that is given $h \in H \backslash\{1\}$, either $h \in P_{H}$ or $h^{-1} \in P_{H}$. If $\exp (h)>0$, then $h \in P_{H}$, otherwise assume $\exp (h) \leq 0$. If $\exp (h)<0$, then $h^{-1} \in P_{H}$, since $\exp \left(h^{-1}\right)=-\exp (h)$. So, assume $\exp (h)=0$. If $n_{h}>0$, then $h \in P_{H}$, otherwise assume $n_{h} \leq 0$. If $n_{h}<0$, then $h^{-1} \in P_{H}$, since $n_{h^{-1}}=-n_{h}$. So, assume $n_{h}=0$. If $h=y^{\epsilon_{1}} z^{\mu_{1}} \ldots y^{\epsilon_{m}} z^{\mu_{m}} \Delta^{n_{h}},-1 \leq \epsilon_{i}, \mu_{i} \leq 1$, satisfies $\exp (h)=n_{h}=0$, then there are two possibilities: either $h=y^{ \pm 1} z^{\mu_{1}} y^{\epsilon_{2}} z^{\mu_{2}} \ldots y^{\epsilon_{m}} z^{ \pm 1}$ or $h=z^{ \pm 1} y^{\epsilon_{2}} z^{\mu_{2}} \ldots y^{\epsilon_{m-1}} z^{\mu_{m-1}} y^{ \pm 1}$. If $h=y^{ \pm 1} z^{\mu_{1}} y^{\epsilon_{2}} z^{\mu_{2}} \ldots y^{\epsilon_{m}} z^{ \pm 1}$, then $h \in P_{H}$. Otherwise, if $h=z^{ \pm 1} y^{\epsilon_{2}} z^{\mu_{2}} \ldots y^{\epsilon_{m-1}} z^{\mu_{m-1}} y^{ \pm 1}$, then $h^{-1}=y^{\mp 1} z^{-\mu_{m-1}} y^{-\epsilon_{m-1}} z^{-\mu_{2}} y^{-\epsilon_{2}} \ldots z^{\mp 1}$, that is $h^{-1} \in P_{H}$.

\section{REFERENCES}

[1] A.L. Agore and G. Militaru, Classifying complements of groups. Applications, to appear Ann. Inst. Fourier, Grenoble.

[2] R. Botto Mura and A.H. Rhemtulla, Orderable groups, Lecture Notes in Pure and Applied Mathematics 27, Marcel Dekker Inc., New York 1977.

[3] M.G. Brin, On the Zappa-Szep Product, Comm. Algebra 33 n.2, (2005), 393-424.

[4] P.F. Conrad, Right-ordered groups, Michigan Math. J. 6 (1959), 267-275.

[5] P. Dehornoy, I. Dynnikov, D. Rolfsen and B. Wiest, Ordering braids, Mathematical Surveys and Monographs 148, American Mathematical Society, Providence, RI, 2008.

[6] B. Deroin, A.Navas, C. Rivas, Groups, Orders and Dynamics, Preprint, ArXiv 1408:5805.

[7] F.W. Levi, Ordered groups, Proc. Indian Acad. Sci. A. 16 (1942), 256-263.

[8] V.M. Kopytov, N. Ya Medvedev, Right-ordered groups, Siberian school of Algebra and Logic, Consultants bureau, New York 1996.

[9] V.M. Kopytov, Free lattice-ordered groups, Algebra Logic 18, n.4 (1979), 259-270.

[10] P.A. Linnell, The space of left orders of a group is either finite or uncountable, Bull.London Math. Soc. 43 (2011), no. 1, 200-202.

[11] D. Rolfsen, Low dimensional topology and ordering groups, Mathematica Slovaca. 64, n.3 (2014), 579600.

[12] A.S. Sikora, Topology on the spaces of orderings of groups, Bull. London Math. Soc. 36 (2004), n.4, 519-526.

[13] J. Szep, On the structure of groups which can be represented as the product of two subgroups, Acta Sci. Math. Szeged 12 (1950), 57-61.

[14] G. Zappa, Sulla costruzione dei gruppi prodotto di due dati sottogruppi permutabili traloro, Atti Secondo Congresso Un. Mat. Ital., Bologna; Edizioni Cremonense, Rome, (1942), 119125.

Fabienne Chouraqui,

University of Haifa at Oranim, Israel.

E-mail: fabienne.chouraqui@gmail.com, fchoura@sci.haifa.ac.il 